\title{
OBSERVATIONS OF BINARY STARS WITH THE DIFFERENTIAL SPECKLE SURVEY INSTRUMENT. V. TOWARD AN EMPIRICAL METAL-POOR MASS-LUMINOSITY RELATION
}

\author{
Elliott P. Horch ${ }^{1,8,9}$, William F. van Altena ${ }^{2}$, Pierre Demarque ${ }^{2}$, Steve B. Howell $^{3,8}$, Mark E. Everett ${ }^{4,8}$, \\ David R. Ciardi ${ }^{5,8}$, Johanna K. Teske ${ }^{6,8}$, Todd J. Henrry ${ }^{7}$, and Jennifer G. Winters ${ }^{7}$ \\ ${ }^{1}$ Department of Physics, Southern Connecticut State University, 501 Crescent Street, New Haven, CT 06515, USA; horche2@ southernct.edu \\ ${ }^{2}$ Department of Astronomy, Yale University P.O. Box 208101, New Haven, CT 06520-8101, USA; william.vanaltena@yale.edu, pierre.demarque@yale.edu \\ ${ }^{3}$ NASA Ames Research Center, Moffett Field, CA 94035, USA; steve.b.howell@nasa.gov \\ ${ }^{4}$ National Optical Astronomy Observatory, 950 North Cherry Avenue, Tucson, AZ 85719, USA; everett@noao.edu \\ ${ }^{5}$ NASA Exoplanet Science Institute, California Institute of Technology, 770 South Wilson Avenue, \\ Mail Code 100-22, Pasadena, CA 91125, USA; ciardi@ipac.caltech.edu \\ ${ }^{6}$ Department of Terrestrial Magnetism and Carnegie Observatories, Carnegie Institute of Washington, \\ 5241 Broad Branch Road, NW, Washington, DC 20015, USA; jteske@ carnegiescience.edu
7 Department of Physics and Astronomy, Georgia State University, Atlanta, GA 30302, USA; thenry@astro.gsu.edu, winters@astro.gsu.edu \\ Received 2014 December 5; accepted 2015 March 1; published 2015 April 14
}

\begin{abstract}
In an effort to better understand the details of the stellar structure and evolution of metal-poor stars, the Gemini North telescope was used on two occasions to take speckle imaging data of a sample of known spectroscopic binary stars and other nearby stars in order to search for and resolve close companions. The observations were obtained using the Differential Speckle Survey Instrument, which takes data in two filters simultaneously. The results presented here are of 90 observations of 23 systems in which one or more companions was detected, and six stars where no companion was detected to the limit of the camera capabilities at Gemini. In the case of the binary and multiple stars, these results are then further analyzed to make first orbit determinations in five cases, and orbit refinements in four other cases. The mass information is derived, and since the systems span a range in metallicity, a study is presented that compares our results with the expected trend in total mass as derived from the most recent Yale isochrones as a function of metal abundance. These data suggest that metal-poor main-sequence stars are less massive at a given color than their solar-metallicity analogues in a manner consistent with that predicted from the theory.
\end{abstract}

Key words: astrometry - binaries: visual - techniques: high angular resolution - techniques: interferometric techniques: photometric

\section{INTRODUCTION}

Torres et al. (2010) have published the most definitive information we have on the mass-luminosity relation (MLR) for main sequence stars to date, but the sample of systems that has yielded the best masses contains only one system with a measured metal abundance $([\mathrm{m} / \mathrm{H}])$ of less than -0.25 . More recent studies using long baseline optical interferometry data have begun to address metallicity (e.g., Boyajian et al. 2012a, 2012b; Freiden \& Chaboyer 2012), but only as low as about $[\mathrm{m} / \mathrm{H}]=-0.5$. Masses and luminosities of metal-poor stars are extremely important to calibrate precisely. For example, the Population II main sequence has been defined by nearby metal-poor stars (e.g., Gratton et al. 1997; Reid 1997), a number of which may be binary. If metal-poor binaries are resolved and individual luminosities can be obtained, these new data could be used to reduce the current scatter in the metal-poor main sequence, allowing for more stringent constraints on stellar models, as well as better ages and distances to galactic globular clusters. The secondary

\footnotetext{
${ }^{8}$ Visiting Astronomer, Gemini Observatory, National Optical Astronomy Observatory, which is operated by the Association of Universities for Research in Astronomy, Inc., under a cooperative agreement with the NSF on behalf of the Gemini partnership: the National Science Foundation (United States), the Science and Technology Facilities Council (United Kingdom), the National Research Council (Canada), CONICYT (Chile), the Australian Research Council (Australia), Ministério da Ciéncia, Tecnologia e Inovañao (Brazil) and Ministerio de Ciencia, Tecnología e Innovación Productiva (Argentina).

9 Adjunct Astronomer, Lowell Observatory.
}

components of metal-poor binaries are especially important in that they will have undergone considerably less change in color and luminosity, and their current observables should thus be close to their zero age locations in the color-magnitude diagram.

Unfortunately, metal-poor systems in the solar neighborhood are less numerous and also typically farther away and therefore fainter than classic Population I systems, often making them difficult objects for optical interferometry. In addition, determining high-quality individual masses is usually timeconsuming and requires both astrometric and spectroscopic observations that span the orbital period. The Differential Speckle Survey Instrument (DSSI) speckle camera combined with the Gemini North telescope provides an excellent opportunity to make quick progress on several low-metallicity systems by combining definitive high-resolution observations obtained at Gemini with spectroscopic data and lower-precision astrometric data taken at other telescopes that are already in the literature.

Direct empirical determinations of individual masses of spectroscopic binaries are possible if the components can be resolved and the separation between the components can be accurately determined. For double-lined systems, the combined spectroscopic/astrometric orbit solution yields individual masses as well as a distance to the system without recourse to parallax measurements; if the distance is independently known, then this can in principle be used to further constrain the mass information. For single-lined systems, an independent 
Table 1

Binary Star Speckle Measures

\begin{tabular}{|c|c|c|c|c|c|c|c|c|}
\hline $\begin{array}{l}\text { WDS } \\
(\alpha, \delta \mathbf{J} 2000.0)\end{array}$ & $\begin{array}{l}\text { HR,ADS } \\
\text { DM, etc }\end{array}$ & $\begin{array}{l}\text { Discoverer } \\
\text { Designation }\end{array}$ & HIP & $\begin{array}{c}\text { Date } \\
\text { (Bess. Yr.) }\end{array}$ & $\begin{array}{c}\theta \\
\left({ }^{\circ}\right)\end{array}$ & $\begin{array}{c}\rho \\
\left({ }^{\prime \prime}\right)\end{array}$ & $\Delta m$ & $\begin{array}{c}\lambda \\
(\mathrm{nm})\end{array}$ \\
\hline \multirow[t]{2}{*}{$00022+2705$} & ADS 17175 & $\mathrm{BU} 733 \mathrm{AB}$ & 171 & 2014.5619 & 339.2 & 0.4016 & 2.64 & 692 \\
\hline & $\ldots$ & $\ldots$ & $\ldots$ & 2014.5619 & 339.3 & 0.4024 & 2.21 & 880 \\
\hline \multirow[t]{4}{*}{$00063+5826$} & HD 123 & STF $3062 A B$ & 518 & 2013.5734 & 353.7 & 1.5411 & $\ldots$ & $692^{\mathrm{a}}$ \\
\hline & $\ldots$ & $\ldots$ & $\ldots$ & 2013.5734 & 353.8 & 1.5395 & $\ldots$ & $880^{\mathrm{a}}$ \\
\hline & $\ldots$ & $\ldots$ & $\ldots$ & 2014.5591 & 355.5 & 1.5479 & $\ldots$ & $692^{\mathrm{a}}$ \\
\hline & $\ldots$ & $\ldots$ & $\ldots$ & 2014.5591 & 355.6 & 1.5552 & $\ldots$ & $880^{\mathrm{a}}$ \\
\hline \multirow{2}{*}{$00089+2050$} & G $131-26$ & BEU 1 & $\ldots$ & 2014.5646 & 94.5 & 0.1458 & 0.77 & 692 \\
\hline & $\ldots$ & $\ldots$ & $\ldots$ & 2014.5646 & 94.8 & 0.1460 & 0.43 & 880 \\
\hline \multirow[t]{2}{*}{$00133+6920$} & GJ 11 & KUI 1 & 1068 & 2014.5646 & 96.4 & 0.8484 & 0.68 & 692 \\
\hline & $\ldots$ & $\ldots$ & $\ldots$ & 2014.5646 & 276.7 & 0.8492 & 0.38 & 880 \\
\hline \multirow[t]{2}{*}{$00325+6714$} & ADS 440 & $\mathrm{MCY} 1 \mathrm{Aa}, \mathrm{Ab}$ & 2552 & 2014.5646 & 222.2 & 0.3703 & 3.05 & 692 \\
\hline & $\ldots$ & $\ldots$ & $\ldots$ & 2014.5646 & 222.2 & 0.3694 & 2.32 & 880 \\
\hline \multirow[t]{4}{*}{$02128-0224$} & HD 13612 & TOK 39Aa,Ab & 10305 & 2013.5734 & 142.3 & 0.0194 & 0.29 & 692 \\
\hline & $\ldots$ & $\ldots$ & $\ldots$ & 2013.5734 & 140.0 & 0.0205 & 0.31 & 880 \\
\hline & $\ldots$ & $\ldots$ & $\ldots$ & 2014.5646 & 127.2 & 0.0193 & 0.51 & 692 \\
\hline & $\ldots$ & $\ldots$ & $\ldots$ & 2014.5646 & 130.9 & 0.0206 & 0.42 & 880 \\
\hline \multirow{2}{*}{$02278+0426$} & HD 15285 & A 2329 & 11452 & 2014.5646 & 347.4 & 0.1442 & 0.09 & 692 \\
\hline & $\ldots$ & $\ldots$ & $\ldots$ & 2014.5646 & 347.5 & 0.1446 & 0.30 & 880 \\
\hline \multirow{2}{*}{$13100+1732$} & HD 114378 & STF 1728AB & 64241 & 2014.5607 & 12.2 & 0.0906 & 0.17 & 692 \\
\hline & $\ldots$ & $\ldots$ & $\ldots$ & 2014.5607 & 12.2 & 0.0908 & 0.26 & 880 \\
\hline \multirow[t]{2}{*}{$14035+1047$} & HD 122742 & GJ 538 & 68682 & 2014.5636 & 76.5 & 0.4052 & 3.80 & 692 \\
\hline & $\ldots$ & $\ldots$ & $\ldots$ & 2014.5636 & 76.7 & 0.4047 & 2.99 & 880 \\
\hline \multirow[t]{2}{*}{$14539+2333$} & GJ 568 & REU 2 & 72896 & 2014.5608 & 92.2 & 0.9591 & 1.36 & 692 \\
\hline & $\ldots$ & $\ldots$ & $\ldots$ & 2014.5608 & 92.5 & 0.9573 & 1.11 & 880 \\
\hline \multirow{4}{*}{$16329+0315$} & HD 149162 & DSG 7 Aa & 81023 & 2013.5615 & 326.7 & 0.0174 & 1.18 & 692 \\
\hline & $\ldots$ & $\ldots$ & $\ldots$ & 2013.5615 & 321.9 & 0.0195 & 1.17 & 880 \\
\hline & $\ldots$ & $\ldots$ & $\ldots$ & 2013.5668 & 124.1 & 0.0069 & 1.37 & 692 \\
\hline & $\ldots$ & $\ldots$ & $\ldots$ & 2013.5668 & 124.1 & 0.0164 & 1.23 & 880 \\
\hline \multirow[t]{4}{*}{$16329+0315$} & HD 149162 & DSG 7 Aa-B & 81023 & 2013.5615 & 227.9 & 0.2824 & 5.63 & 692 \\
\hline & $\ldots$ & $\ldots$ & $\ldots$ & 2013.5615 & 226.5 & 0.2841 & 4.85 & 880 \\
\hline & $\ldots$ & $\ldots$ & $\ldots$ & 2013.5668 & 227.5 & 0.2881 & 5.33 & 692 \\
\hline & $\ldots$ & $\ldots$ & $\ldots$ & 2013.5668 & 228.5 & 0.2824 & 3.84 & 880 \\
\hline \multirow[t]{4}{*}{$17080+3556$} & ADS 10360 & HU 1176AB & 83838 & 2013.5642 & $\ldots$ & $\ldots$ & 0.30 & $692^{\mathrm{b}}$ \\
\hline & $\ldots$ & $\ldots$ & $\ldots$ & 2013.5642 & $\ldots$ & $\ldots$ & 0.31 & $880^{\mathrm{b}}$ \\
\hline & $\ldots$ & $\ldots$ & $\ldots$ & 2014.5471 & $\ldots$ & $\ldots$ & 0.40 & $692^{\mathrm{b}}$ \\
\hline & $\ldots$ & $\ldots$ & $\ldots$ & 2014.5471 & $\ldots$ & $\ldots$ & 0.30 & $880^{\mathrm{b}}$ \\
\hline $17247+3802$ & BD+38 2932 & HSL $1 \mathrm{Aa}, \mathrm{Ab}$ & 85209 & 2013.5643 & 74.3 & 0.0050 & 0.58 & 692 \\
\hline & $\ldots$ & $\ldots$ & $\ldots$ & 2013.5643 & 74.3 & 0.0044 & 0.17 & 880 \\
\hline & $\ldots$ & $\ldots$ & $\ldots$ & 2014.5471 & 53.0 & 0.0233 & 0.13 & 692 \\
\hline & $\ldots$ & $\ldots$ & $\ldots$ & 2014.5471 & 51.9 & 0.0232 & 0.19 & 880 \\
\hline $17247+3802$ & BD+38 2932 & HSL $1 \mathrm{Aa}, \mathrm{Ac}$ & 85209 & 2013.5643 & 61.3 & 0.1615 & 2.13 & 692 \\
\hline & $\ldots$ & $\ldots$ & $\ldots$ & 2013.5643 & 61.3 & 0.1649 & 1.71 & 880 \\
\hline & $\ldots$ & $\ldots$ & $\ldots$ & 2014.5471 & 59.4 & 0.2230 & 2.21 & 692 \\
\hline & $\ldots$ & $\ldots$ & $\ldots$ & 2014.5471 & 59.6 & 0.2226 & 1.92 & 880 \\
\hline $18099+0307$ & ADS 11113 & YSC 132Aa,Ab & 89000 & 2013.5643 & 35.1 & 0.0156 & 0.20 & 692 \\
\hline & $\ldots$ & $\ldots$ & $\ldots$ & 2013.5643 & 36.4 & 0.0149 & 0.01 & 880 \\
\hline & $\ldots$ & $\ldots$ & $\ldots$ & 2014.5636 & 92.6 & 0.0205 & 0.00 & 692 \\
\hline & $\ldots$ & $\ldots$ & $\ldots$ & 2014.5636 & 91.0 & 0.0204 & 0.29 & 880 \\
\hline $19027+4307$ & HD 177412 & YSC 13 & 93511 & 2014.5640 & 247.9 & 0.0372 & 0.83 & 692 \\
\hline & $\ldots$ & $\ldots$ & $\ldots$ & 2014.5640 & 248.0 & 0.0382 & 0.90 & 880 \\
\hline $19264+4928$ & GJ 1237 & YSC 134 & 95575 & 2013.5674 & 341.5 & 0.0209 & 0.25 & 692 \\
\hline & $\ldots$ & $\ldots$ & $\ldots$ & 2013.5674 & 159.1 & 0.0254 & 0.48 & 880 \\
\hline & $\ldots$ & $\ldots$ & $\ldots$ & 2014.5614 & 289.8 & 0.0222 & 0.39 & 692 \\
\hline & $\ldots$ & $\ldots$ & $\ldots$ & 2014.5614 & 281.2 & 0.0227 & 0.49 & 880 \\
\hline & $\ldots$ & $\ldots$ & $\ldots$ & 2014.5640 & 271.4 & 0.0237 & 0.96 & 692 \\
\hline & $\ldots$ & $\ldots$ & $\ldots$ & 2014.5640 & 272.2 & 0.0225 & 0.71 & 880 \\
\hline $21041+0300$ & HD 200580 & WSI 6AB & 103987 & 2013.5677 & 275.8 & 0.2494 & 1.92 & 692 \\
\hline & $\ldots$ & $\ldots$ & $\ldots$ & 2013.5677 & 275.9 & 0.2484 & 1.79 & 880 \\
\hline & $\ldots$ & $\ldots$ & $\ldots$ & 2014.5645 & 282.0 & 0.2561 & 1.84 & 692 \\
\hline & $\ldots$ & $\ldots$ & $\ldots$ & 2014.5645 & 282.3 & 0.2555 & 1.75 & 880 \\
\hline $21041+0300$ & HD 200580 & DSG 6Aa,Ab & 103987 & 2013.5677 & 242.0 & 0.0151 & 1.18 & 692 \\
\hline & $\ldots$ & $\ldots$ & $\ldots$ & 2013.5677 & 245.6 & 0.0188 & 1.77 & 880 \\
\hline & $\ldots$ & $\ldots$ & $\ldots$ & 2014.5645 & 216.1 & 0.0302 & 1.94 & 692 \\
\hline
\end{tabular}


Table 1

(Continued)

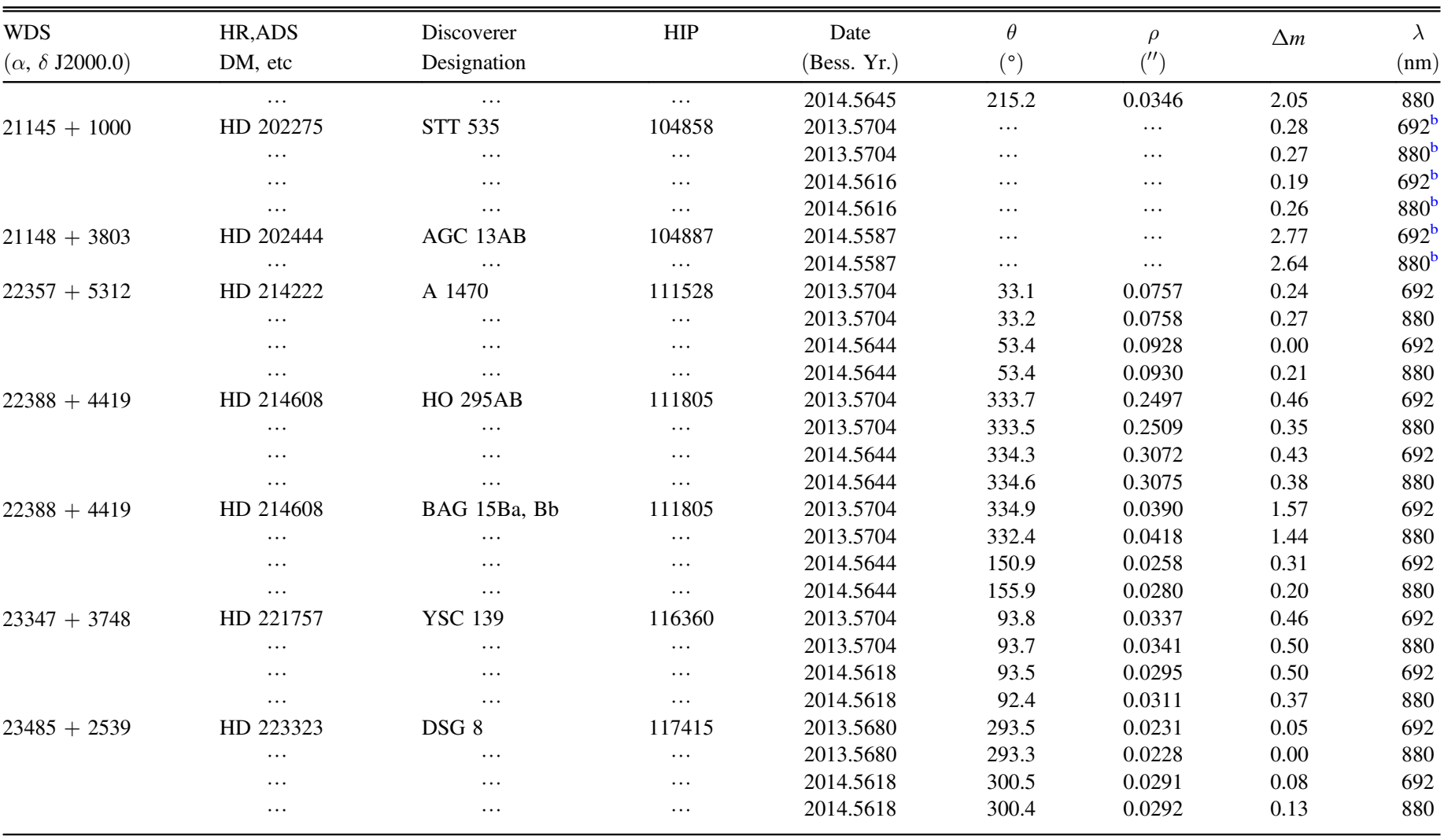

Notes.

${ }^{\text {a }}$ Photometry for this observation does not appear because the $q^{\prime}$ factor discussed in the text was above $0.6 \operatorname{arcsec}^{2}$.

${ }^{\mathrm{b}}$ Astrometry for this observation does not appear because it was used in the determination of the scale.

distance measure is needed to complete the path to individual masses. With a spectroscopic orbit and parallax in hand, even two or three resolved observations spread out along the orbit can be used to reliably measure the semimajor axis and inclination and therefore provide the basis for mass determinations.

The observations presented in this paper include stars that span a range of metal abundance from approximately the solar value to $[\mathrm{m} / \mathrm{H}]=-1.39$ with roughly one third having $[\mathrm{m} / \mathrm{H}]$ values in the range from -0.4 to -1.39 . The spectral types for the sample as a whole range from early-F to early-K. We selected the list of targets from two main sources: the sample of double-lined spectroscopic binaries appearing in the GenevaCopenhagen spectroscopic survey of nearby stars (Nordström et al. 2004), and the spectroscopic survey of proper motion stars of Carney, Latham, and their collaborators (e.g., Goldberg et al. 2002 and references therein). We looked for stars that also had revised Hipparcos parallaxes (van Leeuwen 2007) and roughly prioritized the resulting subset by a combination of distance and metal abundance. Most of the systems that we observed already have spectroscopic orbits. With the astrometry obtained in the work described here, we can derive mass information and constrain stellar structure theory; in particular, the dependence of mass on metallicity for a given spectral type (or equivalently, effective temperature) on the main sequence. While our ultimate goal is to provide a high-precision empirical calibration of the low-metallicity MLR and to use the most relevant systems to obtain a detailed understanding of metal- poor stellar evolution, the current work centers on the identification of some of those systems most likely to provide important information and a preliminary study of the trend in mass with metallicity.

\section{OBSERVATIONS AND DATA REDUCTION}

The DSSI (Horch et al. 2009) was first used at the Gemini North telescope in July of 2012. Results of those observations are found in Horch et al. (2012), Howell et al. (2012), and Horch et al. (2014). In July of 2013 and 2014, DSSI enjoyed official visiting instrument status at the Gemini North, where it was made available to the community, and several scientific programs were executed in each of those two summers.

The instrument records speckle patterns in two filters simultaneously. We refer to the two channels of the instrument as the reflective and transmissive channels, depending on whether the light detected has been transmitted through the dichroic element or if it has been reflected off of it. In the case of all observations here, the reflective channel recorded data through a filter with a center wavelength of $880 \mathrm{~nm}$ and a $50 \mathrm{~nm}$ FWHM transmission, and the transmissive channel recorded data through a $692 \mathrm{~nm}$ center-wavelength filter with a $40 \mathrm{~nm}$ FWHM. These filters were chosen to maximize the limiting magnitude and overall data quality obtained with the instrument. Given that the effects of atmospheric turbulence are less severe at redder wavelengths, these filters allow us to use longer frame integration times on each speckle pattern. Atmospheric dispersion is less of 
an issue in the red, so the use of these filters permits high-quality observing at larger airmass. The two wavelengths are also sufficiently separated to give color information of the components of the binary systems that we observe.

During the 2013 and 2014 runs at Gemini, we were able to obtain observations of a number of binaries drawn from the lists discussed above, as well as to search for close companions to several nearby stars in other cases. This paper is mainly focused on the results of the former group, although it was sensible to include the latter one as the data reduction and analysis techniques were identical. In both cases, Gemini's large aperture allows DSSI to obtain extremely high-resolution images owing to the smaller diffraction limit of the telescope relative to most other speckle programs in operation today, as well as the ability to successfully observe relatively faint sources that in many cases are not easily observable at this time using long-baseline optical interferometry.

\subsection{Reduction Method}

The reduction scheme for binary star observations with DSSI has been described in other papers, most recently in Horch et al. (2011a) and Horch et al. (2012). For the observations discussed here, the typical observation consisted of a sequence of $100060 \mathrm{~ms}$ exposures recorded in each channel of the instrument simultaneously. These are stored as separate FITS stacks, where each frame has a format of $256 \times 256$ pixels. The reduction consists of (1) forming the autocorrelation of each frame and summing these over the 1000 frame stack and (2) computing the so-called "near-axis" subplanes of the image bispectrum for each observation. A reconstructed image is then formed by dividing the Fourier transform of the autocorrelation of the binary with that of the point source, taking the square root, and then combining that with a phase function derived from the bispectral subplanes using the method of Meng et al. (1990). (The point source data are obtained by observing a bright, unresolved star.) That results in an estimate of the Fourier transform of the true, diffraction-limited source intensity distribution. It is low-pass Gaussian filtered and inverse transformed to arrive at the reconstructed image. The reconstructed image of an observation is the primary data product that we use for determining if a companion is present. If no companions are seen, then we use the reconstruction to derive the detection limits for the observation. If at least one companion is detected, then in order to obtain the astrometry and photometry relative to the primary star, we use the power spectrum, where we perform a weighted least-squares fit to a cosine squared function, that is, the fringe pattern seen in the Fourier plane.

For the 2014 run, we developed a program that would allow us greater flexibility in the choice of a point source and greater efficiency while observing. Point sources have generally been necessary to observe close in time and close in sky position to our science targets in order to have a "real time" point-spread function that matches the observing conditions of the science target for our deconvolution process. (This is especially true at airmasses above 1.4.) Recognizing that the details of the pointspread function are mainly due to residual atmospheric dispersion and therefore related to the sky position of the source at the time of the observation (altitude and azimuth), the new program takes as input a point source observed at very high elevation (i.e., one with little dispersion) and builds in the expected dispersion for the sky position of the science target. We compared the results from both point sources taken near in time and near in sky position to the science target versus those from point sources made in this way, and found no significant difference in the quality of the astrometry and photometry. Generally speaking, for the objects shown in Table 1, we used unmodified point sources for most of the 2013 observations and high-elevation point sources modified by the program for the 2014 observations.

\subsection{Pixel Scale and Orientation}

The pixel scale and orientation were determined using the same method that was used in our first experience with DSSI at Gemini (Horch et al. 2012). While our preferred method would have been to use a slit mask mounted in the converging beam of the telescope as we have done at WIYN, the impracticality of mounting and unmounting such a mask at Gemini as well as a desire to make the science observing as efficient as possible have led us to the use of calibration binaries to derive the pixel scale. For the present work, we selected three bright binaries with extremely high-quality orbits appearing in the Sixth Catalog of Visual Orbits of Binary Stars (Hartkopf et al. 2001a). These were HIP $83838=$ HU 1176AB, HIP $104858=$ STT 535, and HIP $104887=$ AGC 13AB. We observed each with the instrument, reduced the data in the manner described in the previous subsection, and compared the location of the secondary in the resulting data with the ephemeris positions in each case.

From our long observing program with DSSI at the WIYN telescope $^{10}$ with DSSI (2008-2013), we know that there is a small amount of distortion in the reflective channel; we have been able to map this out extensively at WIYN, and it has remained essentially constant throughout the years of use at that telescope. The position angles of the binaries used in the scale calibration for the current work allowed us to determine that the effect was consistent with the WIYN distortion model; we therefore assumed that model and then calculated the final position angles and separation for the calibrators based on that. We then obtained scale values of 0.0108 arcsec per pixel in the transmissive channel of the instrument $(692 \mathrm{~nm})$ and 0.0114 arcsec per pixel in the reflective channel $(880 \mathrm{~nm})$. Using the published uncertainties in the orbital elements and our own measurement uncertainties as discussed below, we estimate that these values are uncertain at the level of approximately $\pm 0.1 \%$. Likewise, the chip orientation is determined to within about \pm 0.2 . Given that the speckle images had a format of $256 \times 256$ pixels, the field of view was therefore about $2.8 \times 2.8$ arcsec.

\section{RESULTS}

Table 1 shows the main results of the observations. The columns give: (1) the Washington Double Star (WDS) number (Mason et al. 2001a), which also gives the right ascension and declination for the object in 2000.0 coordinates; (2) a secondary identifier, most often the Henry Draper Catalogue (HD) number for the object; (3) the Discoverer Designation; (4) the Hipparcos Catalogue number (ESA 1997); (5) the Besselian date of the observation; (6) the position angle $(\theta)$ of the secondary star relative to the primary, with north through east defining the positive sense of $\theta ;(7)$ the separation of the

\footnotetext{
${ }^{10}$ The WIYN Observatory is a joint facility of the University of WisconsinMadison, Indiana University, the National Optical Astronomy Observatory, and the University of Missouri.
} 

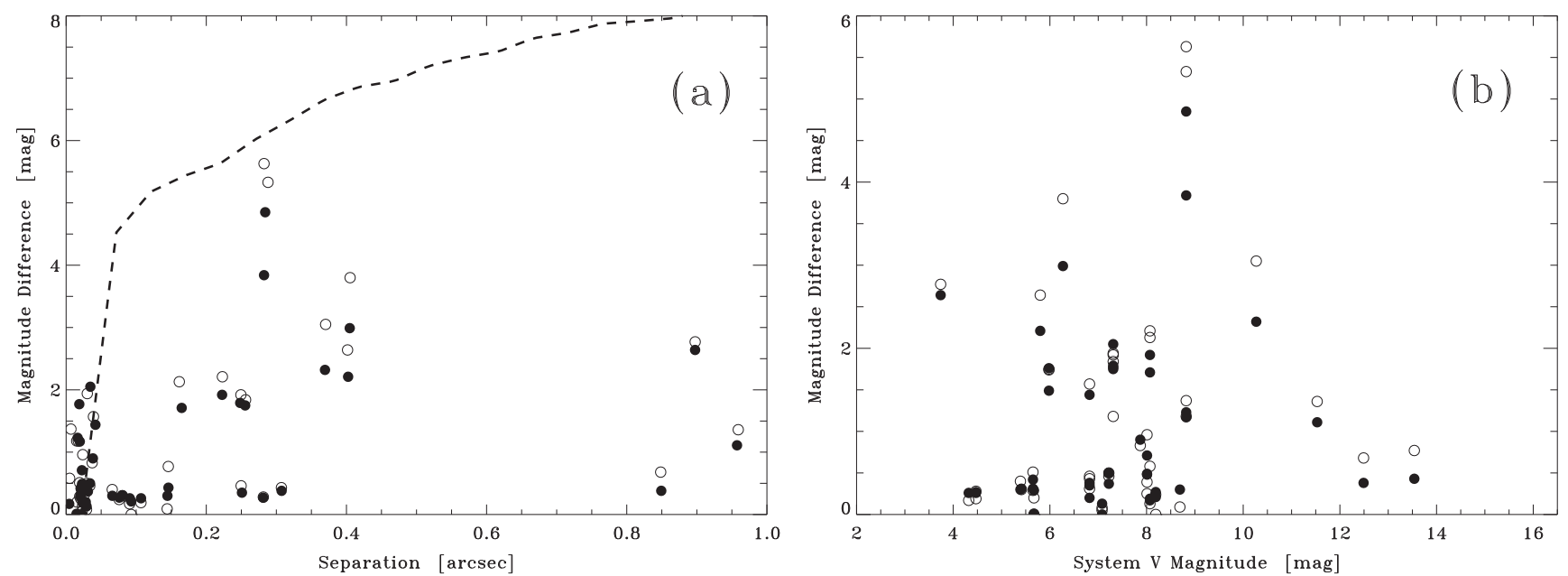

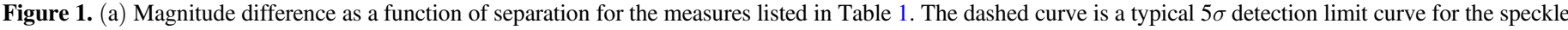

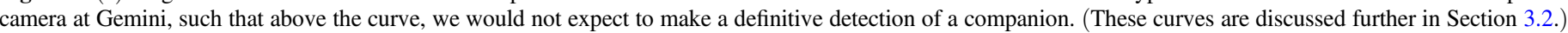

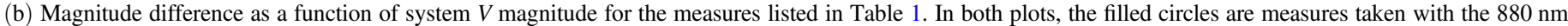
filter and open circles are measures in the $692 \mathrm{~nm}$ filter.

two stars $(\rho)$, in arcseconds; (8) the magnitude difference $(\Delta m)$ of the pair; $(9)$ center wavelength of the filter used; and (10) FWHM of the filter transmission in nanometers. Position angles have not been precessed from the dates shown and are left as determined by our analysis procedure, even if inconsistent with previous measures in the literature. Two objects have no previous detection of the companion; we suggest discoverer designations of DSG (DSSI-Gemini) 7 and 8 , and will refer to them as such throughout the rest of this paper. DSG 7 is in fact a triple system, with the third, wider component having a magnitude difference from the primary of over 5 magnitudes in the $692 \mathrm{~nm}$ filter.

To give some feel for the basic properties of the sample of stars appearing in Table 1 overall, we show in Figure 1(a) the magnitude difference as a function of the separation of the component from the primary star. The majority of observations are clustered at very small separations; these measurements would be difficult to obtain at smaller telescopes. The dashed curve shown is an average-quality $5 \sigma$ detection limit curve for DSSI at Gemini for the $692 \mathrm{~nm}$ filter. These curves are determined by studying the statistics of local peaks in the reconstructed images we obtain; more about how it is calculated will be discussed in Section 3.2. In Figure 1(b), we plot the magnitude difference observed as a function of the total (system) apparent $V$ magnitude for the binary. This shows that the stars we have observed so far for this project have magnitudes in the range $6<V<10$, although it would be possible to observe much fainter sources at Gemini at high signal-to-noise; we plan to include fainter targets for this work in the future.

In Figure 2, we show contour plots of the reconstructed images obtained for the triple system HIP 111805. The secondary star is itself a double-lined spectroscopic binary of period 551 days that has been sporadically detected at the diffraction limit of the $6 \mathrm{~m}$ Special Astrophysical Observatory telescope by Balega et al. (2002, 2006, 2007). Panels (a) and (b) of the figure show the images at $692 \mathrm{~nm}$ and $880 \mathrm{~nm}$ respectively obtained in 2013 July, and panels (c) and (d) show the same for the 2014 July data. The asymmetric elongation of the secondary reveals that it is in fact a binary of modest magnitude difference itself and that it has separation near the diffraction limit of the telescope. The position angle of this system has changed by nearly $180^{\circ}$ between the two observation epochs. This system will be discussed further in Section 4.

\subsection{Relative Astrometry and Photometry}

To characterize the precision of the relative astrometry, we first compared the results obtained in the two channels of the instrument for the same observation by forming the differences between the two channels for position angle and separation. These are shown in Figure 3. Considering only observations with separations from 0.0215 to 1.0 arcsec, we obtain an average difference in position angle of $0.24 \pm 0.37$. For the separation values, the average difference is $-0.34 \pm 0.33$ mas. These values indicate that there is no measurable systematic error in the scale or orientation values applied to the data. The standard deviation of the position angle differences is $1: 99 \pm 0.26$, while for separation, we obtained a value of $1.79 \pm 0.24$ mas. Since we are forming a difference between two measures of presumably the same uncertainty, these values will be $\sqrt{2}$ larger than the intrinsic repeatability of an individual measure. Therefore, we judge that, on average, the values in Table 1 have an intrinsic precision of approximately $1.41 \pm 0.18$ in position angle and $1.27 \pm 0.17$ mas in separation. These numbers would be reduced by another factor of $\sqrt{2}$ by averaging the astrometric results in both channels. While this was not done in Table 1, if one did take that step, the values would be reduced to $1.00 \pm 0.13$ and $0.90 \pm 0.12$ mas. These are very much in line with the values obtained for the earlier observations published in Horch et al. (2012). The precision of the position angle is a function of separation, and degrades as the linear scatter subtends a larger angle and the separation becomes smaller. Our measures have median separation of $\sim 0.1$ arcsec, so that the angular uncertainty will be dominated by the half of our measures below this, down to the diffraction limit. Taking an average separation about 0.05 arcsec for these objects, we would expect them to have an angular uncertainty of $\arctan (0.00127 / 0.05)=1.5$, based on the linear uncertainty value. This is consistent with the angular uncertainty derived above. 

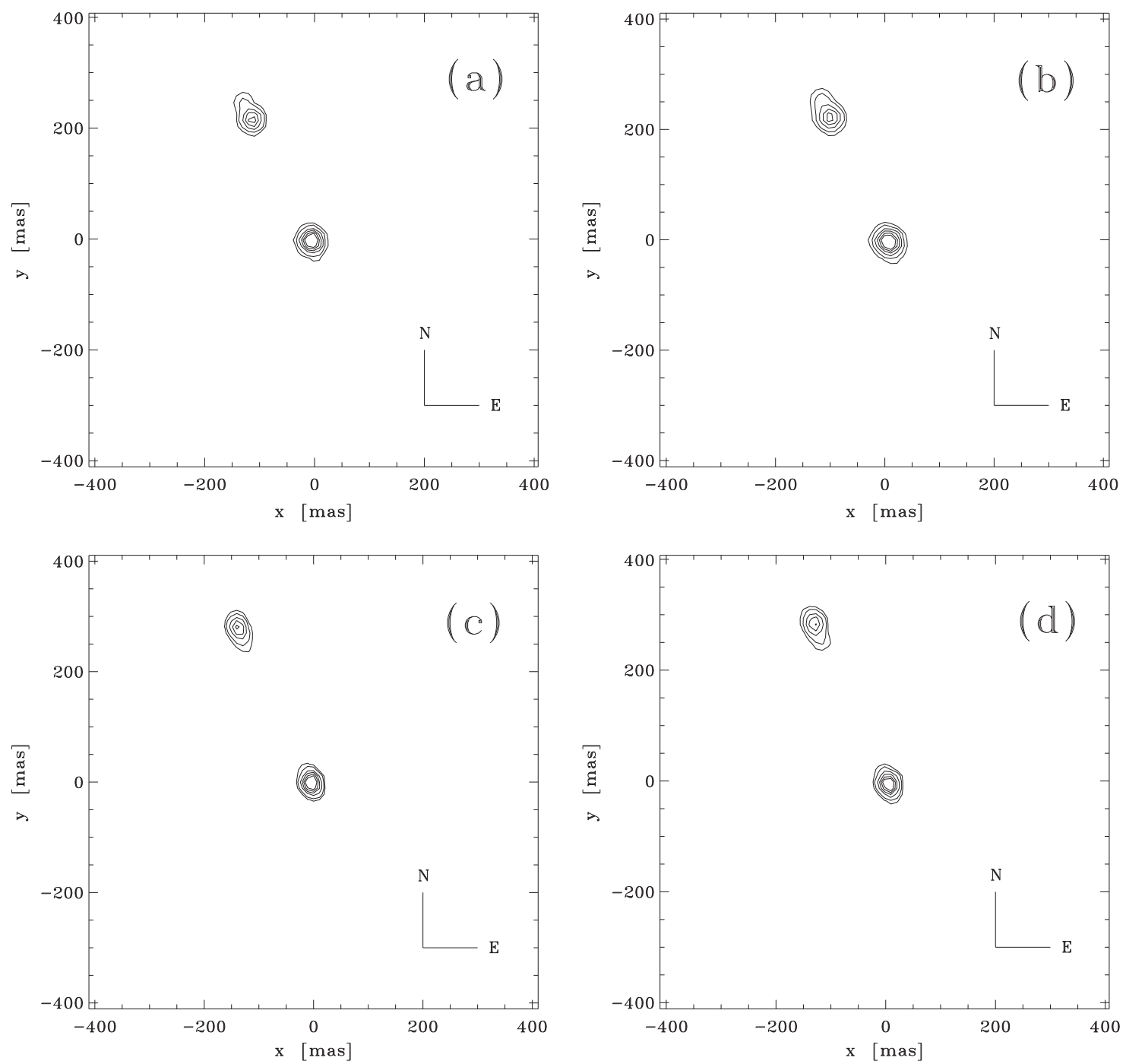

Figure 2. Contour plots of reconstructed images for HIP 111805, a triple system where the secondary is a known spectroscopic binary with period 551.6 days. Contours are drawn at $0.05,0.1,0.2,0.3,0.4$, and 0.5 of the maximum of each array (the central peak corresponding to the primary star). The images labeled (a) and (b) are the 692- and $880 \mathrm{~nm}$ images from 2013 July 27 respectively, and the images labeled (c) and (d) are the same for the observation of 2014 July 25 . The strong asymmetry shape of the contours of the secondary star indicates that it is itself binary, and that the position angle has changed by approximately $180^{\circ}$ from 2013 to 2014.
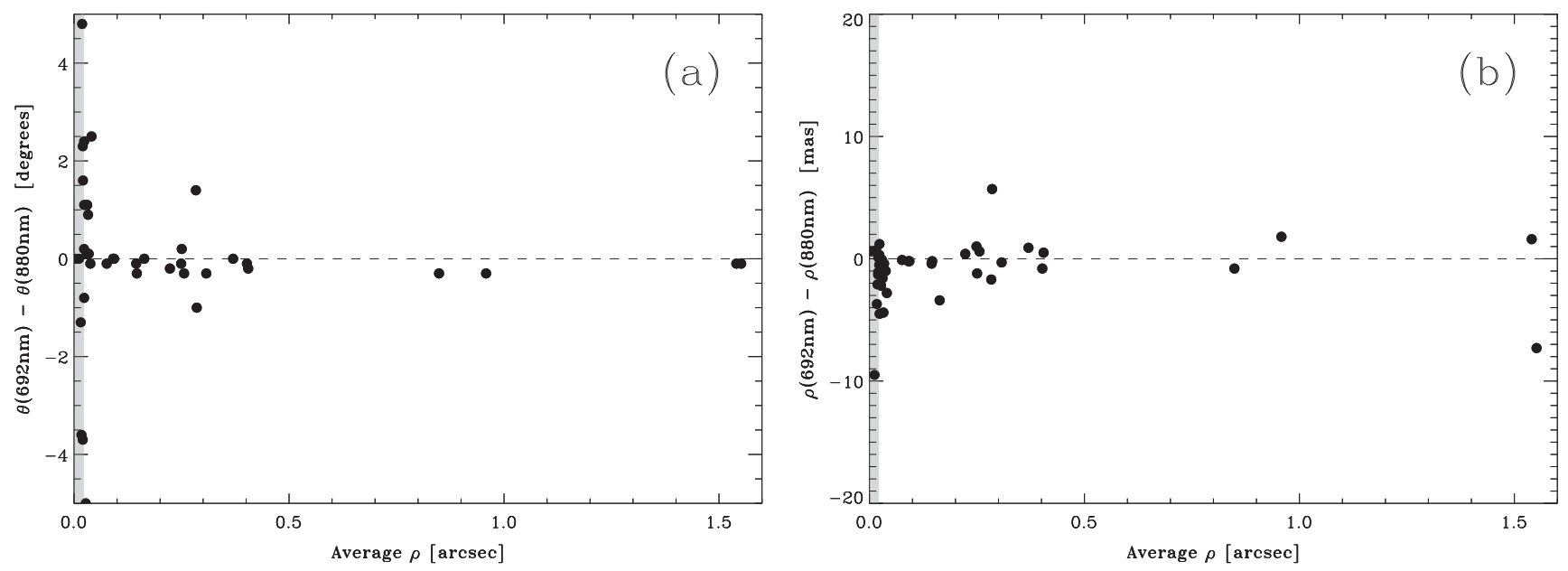

Figure 3. Measurement differences between the two channels of the instrument plotted as a function of measured separation, $\rho$. (a) Position angle $(\theta)$ differences. (b) Separation $(\rho)$ differences. In both plots, the gray band at the left marks the region below the diffraction limit of the telescope. 

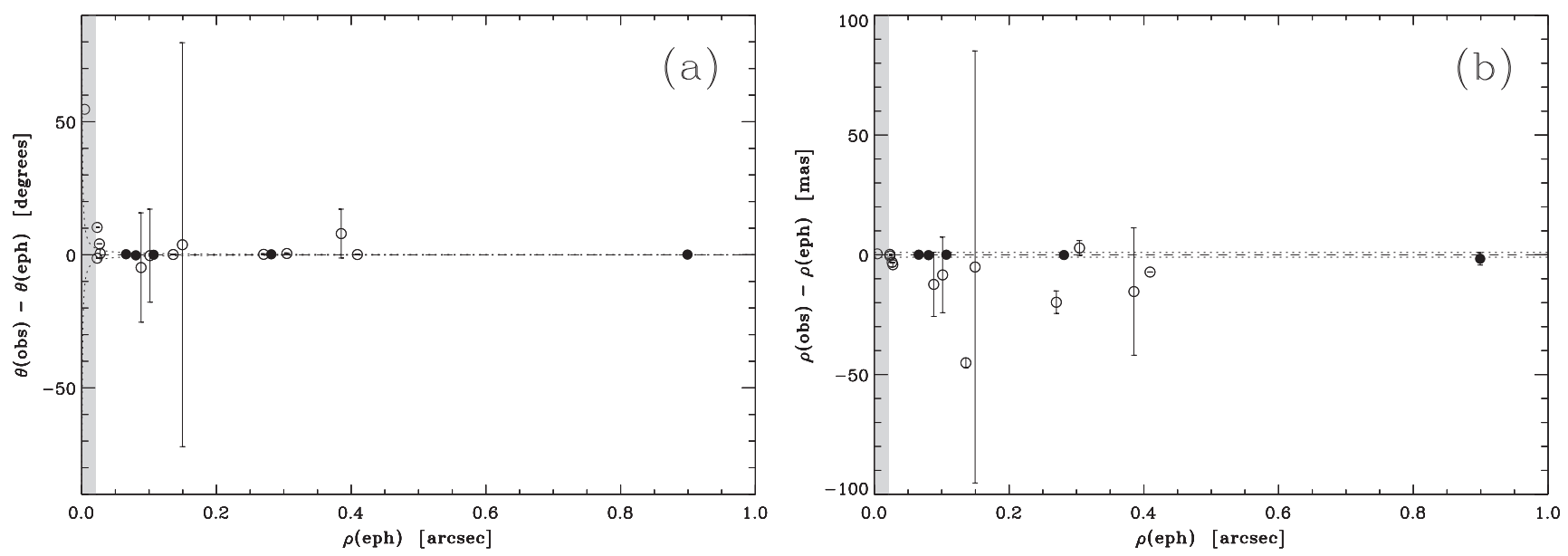

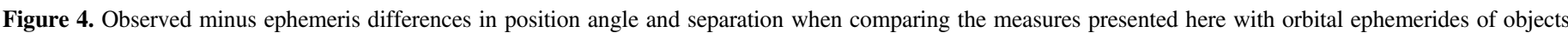

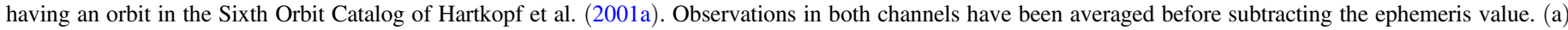

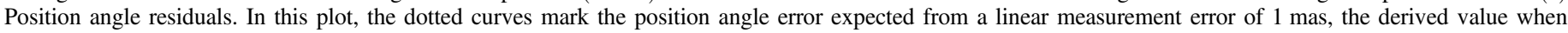

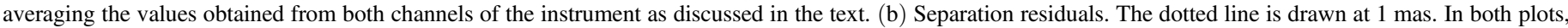

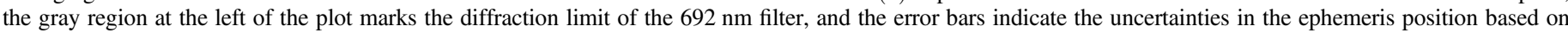

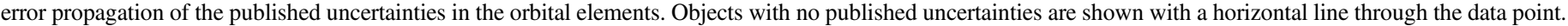
Filled circles indicate the objects used in the scale calibration.

Some measures in Table 1 have separations below the diffraction limit. We have discussed this type of situation in Horch et al. (2006a) and Horch et al. (2011b), where we find in the latter reference that comparing the results in the two channels of the instrument allows us the ability to distinguish between elongation of speckles due to residual atmospheric dispersion and that due to the presence of an unresolved companion. For the measures below the diffraction limit in Table 1, the consistency in the separation determination between both channels of the instrument gives good that we are indeed measuring the presence of an unresolved companion. Because the speckles from the primary and secondary stars are cases are blended in these cases, there is some loss of precision in the measures we obtain; for example, at WIYN, the uncertainty in separation roughly doubled for pairs observed below the diffraction limit with DSSI. While we do not yet have enough measurements to characterize this at Gemini, it would seem to be a reasonable assumption that the same is true at the larger aperture.

A number of systems in Table 1 have a previous orbit determination listed in the Sixth Orbit Catalog (Hartkopf et al. 2001a). We have computed the ephemeris positions of these systems for the epoch(s) of observation shown in Table 1, and compared the separations and position angles to what we obtained. These results are shown in Figure 4. In some cases, the orbital elements are published with uncertainties; in these cases, we can compute uncertainties in the ephemeris positions themselves, and where ever possible, these have been included in the figures.

Especially in the separation plot, there are three data points that deviate significantly from the zero line. These are STF 1728 (at ephemeris separation 0.1358 arcsec) and the two observations of $\mathrm{HO} 295$ (at separations of 0.2701 and 0.3045 arcsec respectively). It is not clear at this point why the deviation of STF 1728 is so great. The orbit is relatively recent and of excellent quality (Muterspaugh et al. 2010). However, the next periastron passage is predicted to be in April of 2015 , and the motion is already relatively fast at this point. We have recomputed the orbit based on data from 1994 to the present (and including the points in Table 1), and we find that the data are consistent with a slightly shorter period $(25.84 \mathrm{yr}$ versus 25.97 , and a slightly different time of periastron passage (2015.11 versus 2015.31), and with all other orbital elements similar to the Muterspaugh et al. orbit. We suggest that if further observations of this system can be made over the next year as the system goes through periastron, then it may be sensible to revise the orbit at that point. The deviation of the HO 295 points is explainable considering that this is a triple system (as shown in Figure 2), and the current orbit for the AB pair is now almost 20 years old (Hartkopf et al. 1996). In the next section, we present new orbital elements for this system. Taking these exceptions into account, the data overall suggest that, once again, there is no evidence for a systematic error in the scale and orientation.

Turning now to the photometry, our previous papers (e.g., Horch et al. 2011a and references therein) have discussed the importance of establishing the ratio of the separation to the size of the isoplanatic patch in order to have confidence in the differential photometry obtained in speckle observations. As the isoplanatic patch is inversely proportional to the seeing, a proxy parameter which we have called $q^{\prime}$ can be established as the seeing value times the separation of the pair. In general, the magnitude difference would be expected to be close to the true value for low values of $q^{\prime}$, and as $q^{\prime}$ increases, then the $\Delta m$ obtained will be systematically too large, as the decorrelation between the primary and secondary speckle patterns results in a loss of photon correlations at the expected separation.

In Figure 5(a), we plot the magnitude difference we obtain here minus an average value obtained from previous measures appearing in the literature. Specifically, we examined all of the magnitude differences for these systems that exist in the Fourth Interferometric Catalog of (Hartkopf et al. 2001b), and we select only those objects with three or more measures that were obtained with a filter within $20 \mathrm{~nm}$ of $692 \mathrm{~nm}$. These are overwhelmingly dominated by our own measures from the WIYN telescope, which are calibrated in a similar way, and adaptive optics measures of the CHARA group (ten Brummelaar et al. 1996). After removing YSC 134 from consideration 

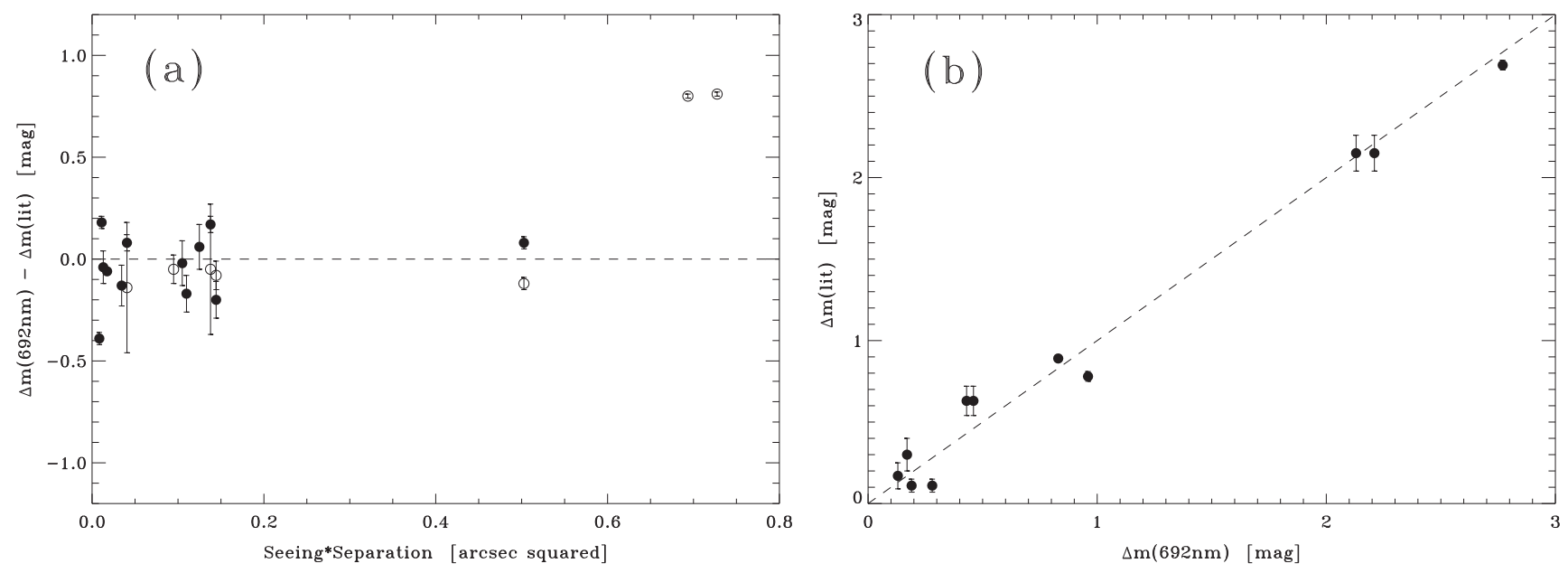

Figure 5. Comparison of the differential photometry presented in Table 1 with existing differential photometry. (a) The difference in $\Delta m$ between our measure at $692 \mathrm{~nm}$ and the $\Delta m$ value appearing in the Hipparcos Catalogue as a function of the parameter $q^{\prime}=$ seeing times separation discussed in the text. Filled circles indicate the average of previous measures in the 4th Interferometric Catalog observed with a similar filter to $692 \mathrm{~nm}$, using the standard error in the mean as the error bar. Open circles show the difference between our $692 \mathrm{~nm}$ result and that appearing in the Hipparcos Catalogue, in the $H_{p}$ filter, with the error bar being the uncertainty shown in the Catalogue. To minimize color effects arising from the difference in filter wavelength between our observations and Hipparcos, only systems with $B-V<0.7$ are included for the Hipparcos comparison. (b) A plot of the $\Delta m$ value as a function of the magnitude difference at $692 \mathrm{~nm}$ in Table 1 for those systems with data in a similar filter in the 4th Interferometric Catalog and $q^{\prime}<0.6$.

as most of its previous measures were obtained below the diffraction limit at WIYN and will therefore be somewhat uncertain, we are left with eleven comparison observations. The result is that, while the diagram is sparsely populated, there is excellent agreement between the literature values and the values in Table 1 for values of $q^{\prime}$ below 0.6. The two points above $q^{\prime}=0.6$ have a larger observed $\Delta m$ than the value appearing in the literature, which is consistent with the loss of correlations due to non-isoplanicity. The mean difference below $q^{\prime}=0.6$ is $0.00 \pm 0.04$ magnitudes, and the standard deviation of these differences is $0.13 \pm 0.03$ magnitudes. Some of this scatter is due to the uncertainty of the literature values themselves; on average, the uncertainty is about 0.07 magnitudes. If we subtract this in quadrature from 0.13 , we obtain an intrinsic repeatability in the magnitude difference of our measures of about 0.11 magnitudes. The comparisons in the plot with Hipparcos values (represented by the open circles) show a slight negative trend; this is expected due to the fact that the Hipparcos filter is considerably bluer than $692 \mathrm{~nm}$. Most of the systems in question are known to be main sequence systems, meaning that the secondary will be redder than the primary, and therefore the system will exhibit a larger magnitude difference in a bluer filter. Figure 5(b) shows a plot of the literature values versus our values for those measures with $q^{\prime}<0.6$; this should be a line of slope one that passes through the origin, and the data are consistent with that from 0 to 3 magnitudes.

\subsection{Non-detections}

Six systems that we observed showed no evidence of a companion to the limit of detection. We show the detection limit in magnitude difference from the primary for both 0.1 and 0.2 arcsec in Table 2. As mentioned earlier, in order to make a determination about the detection limit, we use the reconstructed images obtained from the speckle data reduction. In these images, the primary star is always centered in the image. We investigate the image properties within concentric annuli centered on the primary. Within each annulus, we determine the value of all local
Table 2

High-quality Non-detections and $5 \sigma$ Detection Limits

\begin{tabular}{|c|c|c|c|c|c|c|}
\hline \multirow{2}{*}{$\begin{array}{l}(\alpha, \delta \mathbf{J} 2000.0) \\
\text { (WDS format) }\end{array}$} & \multirow{2}{*}{$\begin{array}{c}\text { Hipparcos } \\
\text { Number }\end{array}$} & \multirow{2}{*}{$\begin{array}{c}\text { Date } \\
\text { (Bess. Yr.) }\end{array}$} & \multicolumn{2}{|c|}{$\begin{array}{l}5 \sigma \text { Det. } \\
\text { Lim., } \\
692 \mathrm{~nm}\end{array}$} & \multicolumn{2}{|c|}{$\begin{array}{l}5 \sigma \text { Det. } \\
\text { Lim., } \\
880 \mathrm{~nm}\end{array}$} \\
\hline & & & $0 . " 1$ & $0 .{ }^{\prime \prime} 2$ & $0 .{ }^{\prime \prime} 1$ & 0.2 \\
\hline $01291+2143$ & 6917 & 2014.5619 & 4.00 & 4.83 & 3.94 & 4.72 \\
\hline $14308+3527$ & 70950 & 2014.5636 & 4.23 & 4.80 & 4.29 & 5.06 \\
\hline $16255+7123$ & 80467 & 2013.5615 & 2.66 & 4.08 & 4.01 & 5.04 \\
\hline $16440+0901$ & 81923 & 2014.5636 & 4.14 & 5.02 & 4.11 & 4.99 \\
\hline $22057+1223$ & 109067 & 2013.5677 & 3.97 & 4.62 & 3.75 & 4.93 \\
\hline $22316+0210$ & 111195 & 2013.5677 & 4.27 & 4.91 & 3.83 & 4.70 \\
\hline
\end{tabular}

maxima. The background level is then set to the average value of these maxima, and a standard deviation of the peak values is computed. The detection limit for that particular annulus is set as the background value plus five standard deviations, that is, it is a $5 \sigma$ limit. A similar calculation is performed on the absolute value of the local minima to make sure that the distributions of maxima and minima are similar. This is then associated with the mean radius of the annulus. Once values for annuli with a range of different separations from the primary have been computed, a cubic spline interpolation is performed to derive the detection limit curve as a function of separation. Generally, these curves have very shallow values of the limiting $\Delta m$ at the smallest separations, a rapid rise leading to a "knee" in the curve at approximately 0.1 arcsec, and a continued slower rise in limiting magnitude out to the largest separations we measure (1.2 arcsec).

Examples of the detection limit curves for one of the objects in Table 2 are shown in Figure 6. This object is actually a known single-lined spectroscopic binary star with a 10 days period, but based on the period, the spectral type of $\mathrm{K} 2.5 \mathrm{~V}$, and the system parallax using the revised Hipparcos value of $42.13 \pm 0.68$ mas (van Leeuwen 2007), we can roughly estimate that the semimajor axis of the orbit is on the order of a few mas; this would not be detectable using DSSI at Gemini even if the magnitude difference were small. The 

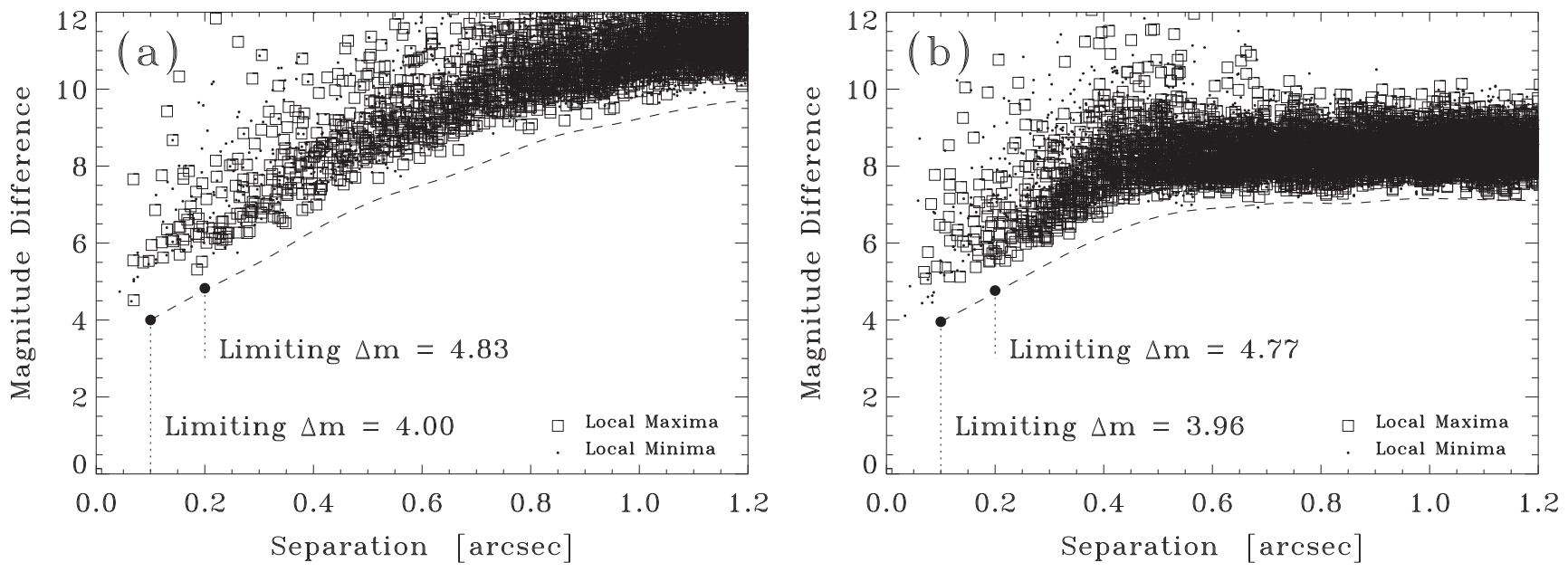

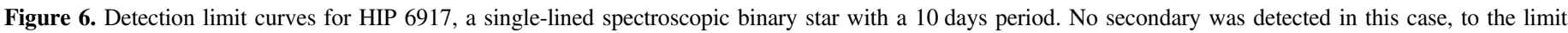
shown in each case. (a) The result for the $692 \mathrm{~nm}$ reconstructed image. (b) The result at $880 \mathrm{~nm}$.

Table 3

Visual Orbit Refinements for Four Systems

\begin{tabular}{|c|c|c|c|c|}
\hline Parameter & HSL $1 \mathrm{Aa}, \mathrm{Ab}$ & YSC 134 & A 1470 & HO $295 \mathrm{AB}$ \\
\hline HIP & 85209 & 95575 & 111528 & 111805 \\
\hline Type of Spectroscopic Orbit & $\mathrm{SB} 2^{\mathrm{a}}$ & $\mathrm{SB} 2^{\mathrm{b}}$ & $\mathrm{SB} 2^{\mathrm{c}}$ & $\mathrm{SB} 2^{\mathrm{d}}$ \\
\hline$P$, years & 1.2283 & 0.45677 & 22.3455 & 29.9995 \\
\hline$i$, degrees & $81.9 \pm 1.8$ & $141.2 \pm 9.2$ & $63.3 \pm 0.3$ & $88.2 \pm 0.1$ \\
\hline$\Omega$, degrees & $56.7 \pm 1.5$ & $29.1 \pm 10.3$ & $110.5 \pm 0.3$ & $154.7 \pm 0.1$ \\
\hline$T_{0}$, years & 1986.3731 & 1990.0465 & 1985.2460 & 1979.8000 \\
\hline
\end{tabular}

Notes.

a The spectroscopic elements are fixed to those of Goldberg et al. (2002).

b The spectroscopic elements are fixed to those of Halbwachs et al. (2012).

c The spectroscopic elements are fixed to those of Pourbaix (2000).

d The spectroscopic elements are fixed to those of Duquennoy (1987).

curves show that there is no other other wider component seen in the vicinity of this star. The other objects in Table 2 had no previous detection of a component either via spectroscopy or high-resolution imaging.

\section{NEW ORBITAL ELEMENTS}

From the objects listed in Table 1, we have selected nine for which the addition of Gemini data permits either an orbit revision or a first orbit determination. All of these systems are spectroscopic binaries, and so, recognizing that in most cases we do not have sufficient astrometry to calculate a good-quality visual orbit, we have fixed the values of period $(P)$, time of periastron passage $\left(T_{0}\right)$, eccentricity $(e)$, and position angle of the node in the plane of the true orbit $(\omega)$ to be those of the spectroscopic orbit prior to fitting for the other three elements, namely semimajor axis $(a)$, inclination $(i)$, and the node $(\Omega)$. The orbit code of MacKnight \& Horch (2004) was then used to determine these three elements and their uncertainties. This code is a grid search of orbital parameters between user-chosen minimum and maximum values, followed by a downhill simplex algorithm to fine-tune the final result. We show our results for the nine systems on which we used this approach in Tables 3 and 4 . Table 3 gives elements for systems that already have an orbit determination in the literature, whereas Table 4 gives the elements for the first orbit determinations. One exception to the method regarding the use of the spectroscopic orbital elements is $\mathrm{BAG} 15 \mathrm{Ba}, \mathrm{Bb}$. In this case, fixing the spectroscopic elements resulted in an orbit with large residuals; we then determined this orbit solely from the relative astrometry, which produced much lower residuals. In studying the difference in the orbital parameters, the time of periastron passage was the only element that was significantly different from those of the spectroscopic orbit. It is not possible to assess whether the value we obtained is consistent with that of the spectroscopic orbit as no uncertainties were given in that case (Duquennoy 1987). In Figure 7, we show four sample orbits of the systems in Tables 3 and 4.

\subsection{Comments on Individual Systems}

\subsubsection{HSL 1}

As discussed in Horch et al. (2006b), this system is a metalpoor hierarchical quadruple system where only the inner three stars have been imaged in the current work due to the very small field of view of the DSSI at Gemini. While data in the literature for the third component of this nearly-edge-on system indicate substantial motion over the last decade that appears highly likely to be orbital in nature, obtaining a definitive orbit 
Table 4

Preliminary Visual Orbits for Five Systems

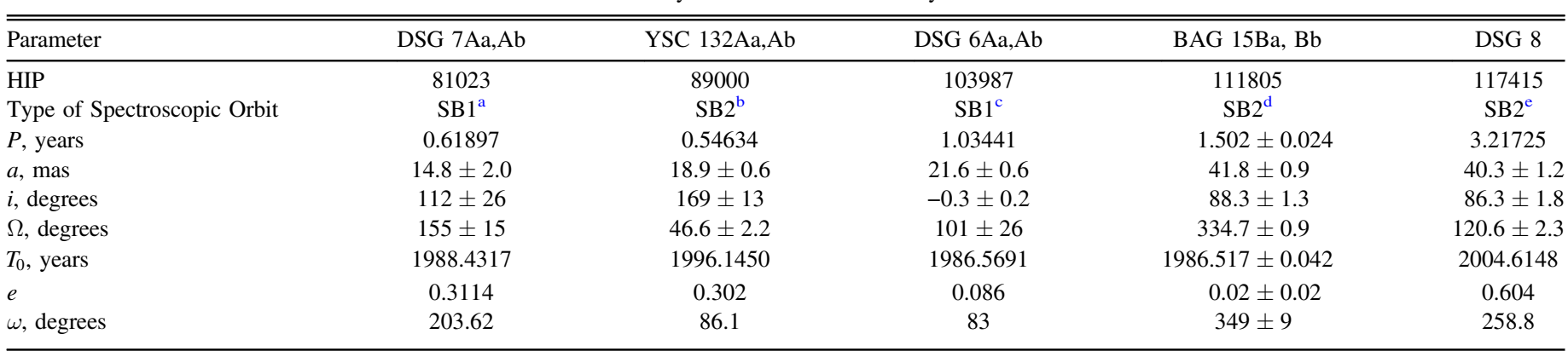

Notes.

${ }^{a}$ The spectroscopic elements are fixed to those of Latham et al. (2002).

b The spectroscopic elements are fixed to those of Griffin (1999).

${ }^{c}$ The spectroscopic elements are fixed to those of Latham et al. (1992).

d All elements are calculated from the speckle data, but a double-lined orbit exists due to Duquennoy (1987). This orbit has similar elements to the orbit here except for the time of periastron passage.

e The spectroscopic elements are fixed to those of Griffin (2007).
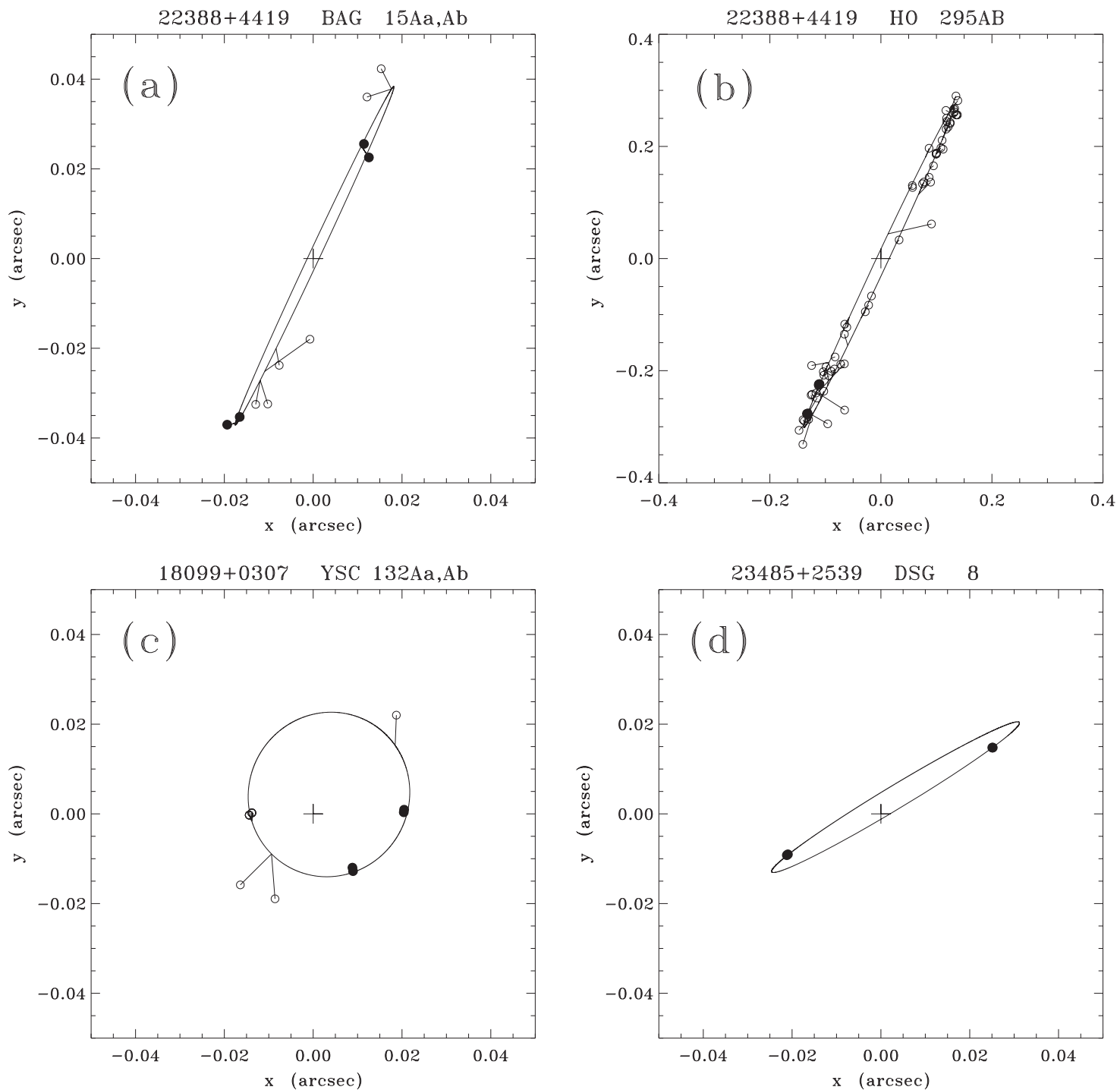

Figure 7. Orbits calculated here for four systems together with data from the literature and our measures from Table 1. The latter are shown with filled circles. All points are drawn with line segments from the data point to the location of the ephemeris prediction on the orbital path. (a) BAG 15Aa,Ab (= HIP 111805), (b) HO $295 \mathrm{AB}$ (= HIP 111805); note that (a) and (b) are a triple system. (c) YSC 132Aa,Ab (= HIP 89000); (d) DSG 8 (= HIP 117415). North is down and east is to the right in all cases. 
will be a difficult proposition until we have almost a full orbit. With less than half the orbit traced out, the period remains uncertain on the level of years, and there is also substantial uncertainty in the semimajor axis.

The Gemini data do however help with the inner pair: they are much more consistent with the visual+spectroscopic orbit in Horch et al. (2006b) than the second orbit calculation there, which is an unconstrained visual orbit that does not use the spectroscopic information. This confirms that the system has an inclination near $90^{\circ}$. Since the inner pair is a double-lined spectroscopic binary, we can independently determine the parallax by comparing the $a \sin i$ value from the spectroscopic orbit (in units of $\mathrm{Gm}$ ) with the value of $a \sin i$ implied from the visual orbital elements (in arcseconds), and in doing this we obtain $22.9 \pm 0.5$ mas. Horch et al. (2006b) computed a value of $23.7 \pm 0.7$, which is consistent with the value here. On the other hand, the revised Hipparcos value of $19.76 \pm 0.82$ mas is almost $4 \sigma$ away from our results; the motion of the third component, which went undetected by Hipparcos, may have some role in this discrepancy.

\subsubsection{YSC 134}

This $\mathrm{K} 2.5 \mathrm{~V}$ system has $[\mathrm{m} / \mathrm{H}]=-0.80$, and is a doublelined system. A visual orbit has recently been computed by Docobo et al. (2013). Their method, like the work here, incorporates the spectroscopic orbital elements, and the orbit is very similar to the one we obtain, although they did not have all of the Gemini data points presented here. Calculating the implied parallax, we obtain $34.9 \pm 6.7$ mas, which is consistent with the Hipparcos revised result of $39.98 \pm 0.73$ mas, within the uncertainty.

\subsubsection{A 1470}

A slightly metal-poor system with $[\mathrm{m} / \mathrm{H}]$ of -0.11 , the spectral type appearing in SIMBAD is that of a G0 subgiant. Using the spectroscopic and visual orbit data, a parallax of $14.07 \pm 0.22$ mas is obtained, which agrees very well with the Hipparcos revised result of $14.15 \pm 0.74$ mas. From this, an absolute $V$ magnitude of 3.94 is obtained. Although the $B-V$ color of the system is consistent with the SIMBAD spectral assignment, the absolute magnitude for the system would seem to be too faint for a subgiant, and more consistent with two slightly later dwarfs. For this reason, we will treat the system as such in the next section.

\subsection{4. $H O 295 A B$ and $B A G 15 B a, B b$}

This is the triple system appearing in Figure 2 and it has $[\mathrm{m} /$ $\mathrm{H}$ ] of -0.29 . The wider component, $\mathrm{HO} 295 \mathrm{AB}$, has a $30 \mathrm{yr}$ period, but is nonetheless known as a spectroscopic doublelined system. Both this component and the smaller-separation component (an SB2 with a $1.5 \mathrm{yr}$ period) are used in the study presented in the next section. Our values of parallax from both orbits are reasonably consistent with Hipparcos: for the inner pair, we have $25.5 \pm 0.5$ mas and for the wider component, we obtain 24.1 mas, though an uncertainty estimate is not possible in this case because the spectroscopic orbit was published without error estimates. The revised Hipparcos parallax is $26.18 \pm 0.60$ mas. Both orbits obtained here are shown in Figure 7.

\subsubsection{DSG 7Aa,Ab}

The measures in Table 1 represent the first resolution of the companion to date. A K0 SB1 with very low metal abundance $([\mathrm{m} / \mathrm{H}]=-1.39)$, this pair has a period of 226 days and a magnitude difference of approximately 1.5. Ideally, one would like to have observations at several different epochs spread out around the orbit, but we have just two observations, both taken in 2013 July. Nonetheless, our measures are consistent with the astrometric orbit in the Hipparcos Catalogue (ESA 1997) in terms of their position angle at the time of observation, and they allow us to determine the size of the orbit to about $13 \%$.

\subsubsection{YSC $132 A a, A b$}

Observations with DSSI at WIYN indicated a companion to this star that was below the diffraction limit of that telescope. The first resolved image came from our 2012 July Gemini run. In 2013 July, the system had moved considerably, and by July of 2014, it was possible to determine the orbit with high reliability, in combination with the spectroscopic information. The orbit of this system is shown in Figure 7.

\subsection{7. $D S G 6 A a, A b$}

This triple system consists of an inner pair which is a singlelined spectroscopic orbit, and wider component first resolved in 1999 by Mason et al. (2001b). Subsequent observations have shown that this wider component does orbit the inner pair, and in fact must have a period of $\sim 20 \mathrm{yr}$. We have made a preliminary orbit calculation that is not presented here; the period and semimajor axis uncertainties led to a large uncertainty in mass so that the system would not yet be useful in the study that we present in the next section. Interestingly, the inclination of that calculation would seem to suggest that the orbit is not coplanar with the spectroscopic orbit, so a full analysis of the system as a whole is warranted. There is archival data from the Fine Guidance Sensors on the Hubble Space Telescope that could be incorporated into a more detailed analysis of both orbits, as well as unpublished WIYN speckle data. However, that effort is beyond the scope of the present paper.

\subsection{8. $D S G 8$}

The measures in Table 1 are the first resolution of this wellknown double-lined spectroscopic binary with period $3.2 \mathrm{yr}$. The observations are separated by nearly $180^{\circ}$, allowing for a very good orbit when combining with the spectroscopic orbital parameters. The orbit is shown in Figure 7. This immediately shows why there have not been previous measures: the system is below the diffraction limit even of Gemini for a large portion of the orbit, due to the inclination. While the spectral type in the literature is F2IV-V, the system is slightly redder than F2; we treat this system as an F5+F5.5 dwarf pair in the next section. The parallax that we can obtain from our SB+visual orbit is in complete agreement with the revised Hipparcos value: $14.3 \pm 0.3$ mas in our case versus $14.51 \pm 0.47$ mas from Hipparcos.

\subsection{Total Mass as a Function of Metallicity}

Table 5 lists some further observed properties of the nine systems identified above. The columns give (1) the discoverer designation; (2) the Hipparcos number; (3) the revised 
Table 5

Further Observed Properties for the Systems in Tables 3 and 4

\begin{tabular}{|c|c|c|c|c|c|c|c|}
\hline Name & HIP & $\begin{array}{c}\pi \\
(\mathrm{mas})\end{array}$ & $\begin{array}{l}\text { Abs. } V \\
\text { Mag. }\end{array}$ & $\begin{array}{l}\text { Spectral } \\
\text { Type }\end{array}$ & {$[\mathrm{m} / \mathrm{H}]$} & $B-V$ & $\begin{array}{c}\Delta m \\
(692 \mathrm{~nm})\end{array}$ \\
\hline HSL $1 \mathrm{Aa}, \mathrm{Ab}$ & 85209 & $19.76 \pm 0.82$ & 4.94 & G5 & $-0.75^{\mathrm{a}}$ & $0.76 \pm 0.02^{\mathrm{b}}$ & $0.36 \pm 0.13$ \\
\hline YSC 134 & 95575 & $39.98 \pm 0.73$ & 6.02 & $\mathrm{~K} 2.5 \mathrm{~V}$ & -0.80 & $0.929 \pm 0.009$ & $0.61 \pm 0.13$ \\
\hline A 1470 & 111528 & $14.15 \pm 0.74$ & 3.94 & G0IV & -0.11 & $0.610 \pm 0.015$ & $0.12 \pm 0.12$ \\
\hline HO $295 \mathrm{AB}$ & 111805 & $26.18 \pm 0.60$ & 3.91 & G0 & -0.29 & $0.581 \pm 0.005$ & $0.45 \pm 0.02$ \\
\hline DSG 7Aa,Ab & 81023 & $23.14 \pm 1.02$ & 5.64 & K0 & $-1.39^{\mathrm{a}}$ & $0.868 \pm 0.004$ & $1.28 \pm 0.10$ \\
\hline YSC $132 \mathrm{Aa}, \mathrm{Ab}$ & 89000 & $21.31 \pm 0.31$ & 2.31 & F5V & -0.13 & $0.490 \pm 0.005$ & $0.07 \pm 0.07$ \\
\hline DSG 6Aa,Ab & 103987 & $19.27 \pm 0.99$ & 3.73 & F9V & -0.51 & $0.547 \pm 0.007$ & $1.54 \pm 0.22$ \\
\hline BAG $15 \mathrm{Ba}, \mathrm{Bb}$ & 111805 & $26.18 \pm 0.60$ & 3.91 & G0 & -0.29 & $0.581 \pm 0.005$ & $0.94 \pm 0.63$ \\
\hline DSG 8 & 117415 & $14.51 \pm 0.47$ & 2.89 & F2IV-V & -0.46 & $0.443 \pm 0.009$ & $0.07 \pm 0.02$ \\
\hline
\end{tabular}

Notes.

${ }^{\text {a }}$ From Latham et al. (1992).

b The Hipparcos $B-V$ has a large uncertainty; we use the value shown in Horch et al. (2006b) here.

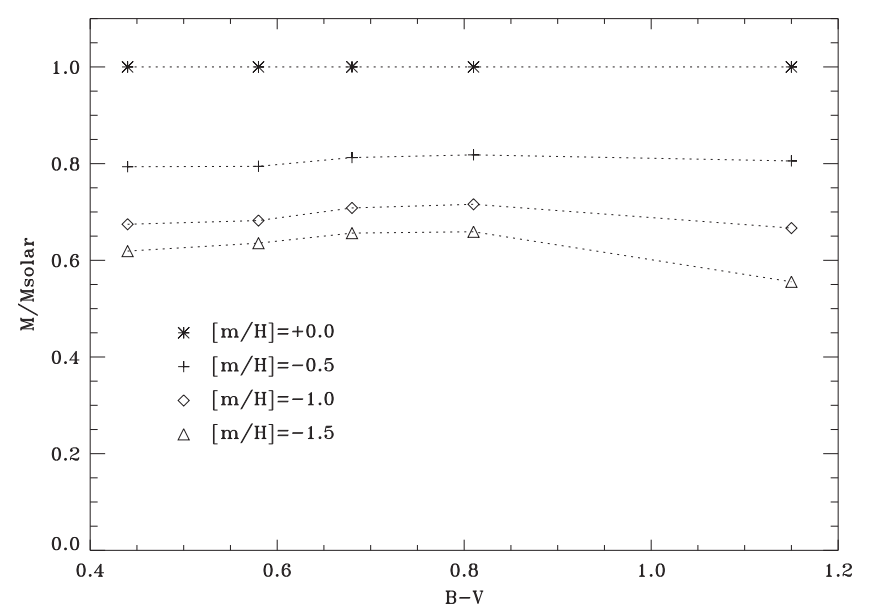

Figure 8. Behavior of stellar mass relative to the solar metallicity value as a function of $B-V$ and metal abundance, as predicted by the Spada et al. (2013) isochrones. This example is for a mixing length parameter of 1.875 and an age of $0.1 \mathrm{Gyr}$, but other ages and mixing lengths give similar results. For a given metal abundance, the mass ratio is relatively uniform across the spectral range of interest.

Hipparcos parallax of van Leeuwen (2007); (4) the absolute magnitude obtained from the apparent magnitude (not listed) and the parallax, where no reddening correction was made since these systems are all nearby; (5) the (composite) spectral type as it appears in SIMBAD; the metal abundance from Holmberg et al. (2009), unless the value is otherwise marked; (6) the $B-V$ value listed in the Hipparcos Catalogue, and (7) the average magnitude difference at $692 \mathrm{~nm}$ from all available DSSI measures of the target. Note that the absolute magnitudes show clearly that all nine systems are on or very near the main sequence.

Using the most recent Yale isochrones (Spada et al. 2013), we investigated the behavior of stellar mass as a function of metal abundance at fixed $B-V$ color. We selected main sequence stars that had spectral types from mid-F to mid-K; this range is similar to the nine systems for which we obtained orbital elements (and hence total masses). The ages we chose were from 0.1 to $4 \mathrm{Gyr}$; this range insures that the spectral types in question will all be close to the main sequence, which we know is the case for all of the systems under study. For a given metal abundance and ranging from $B-V=0.44$ to 1.15 , we then calculated the ratio of the mass extracted from the isochrones to the mass for a star of the same color but with solar metallicity. By definition, this function is one when $[\mathrm{m} / \mathrm{H}]=0.0$, but the isochrones predict that as the metallicity decreases, the ratio also decreases to a value of approximately 0.6 at $[\mathrm{m} / \mathrm{H}]=-1.5$. While there is some variation depending on age and the choice of the mixing length parameter, we found that there was little dependence on a star's color (or equivalently, spectral type) over the range of interest here, as shown in Figure 8. Since the curves are nearly independent of spectral type, it should be true that the total mass of a binary star will follow the same trend, provided that both stars fall in the spectral range of the simulations. We also found the same result with the older Yale-Yonsei isochrones found in Demarque et al. (2004).

In Tables 3 and 4, we have dynamical estimates of the total mass of nine systems that span a considerable range in metal abundance. If we could estimate the mass of the solarabundance analog for the system, then we could compute the ratio and examine whether the trend is similar to what the isochrones predict. To make this mass estimate, we use the composite $B-V$ color for the system as it appears in the Hipparcos Catalogue and the average magnitude difference measured by DSSI at $692 \mathrm{~nm}$, combining all observations of the system in that filter to date at Gemini, i.e., the last two columns of Table 5. We then use the solar-abundance spectral library of Pickles (1998) to combine stars of different spectral types to produce a composite $B-V$ value and $\Delta m$ at $692 \mathrm{~nm}$ that is as close as possible to the measured values. We incorporate into these models a standard atmospheric transmission curve, the known filter transmission curve, and dichroic transmission curve for DSSI. We consider only the 692- and not the $880 \mathrm{~nm}$ data at this stage because we have a more reliable transmission curve for this filter. After giving the same two columns to identify the objects as in Table 5, Table 6 shows in the third and fourth columns the assigned component spectral types and the composite $B-V$ that would be obtained. The latter should be directly comparable to the next-to-last column of Table 5. Figure 9(a) shows that the scatter is modest; the standard deviation of the difference between the measured and simulated colors is less than 0.03 magnitudes. Figure 9(b) shows a similar result for the simulated $\Delta m$ values at $692 \mathrm{~nm}$; here, the uncertainty is dominated by the measured values and not the simulation.

We can then convert these assigned spectral types into mass estimates; for this we used the standard reference of SchmidtKaler (1982), and these appear in Columns 6 and 7 of Table 6. 

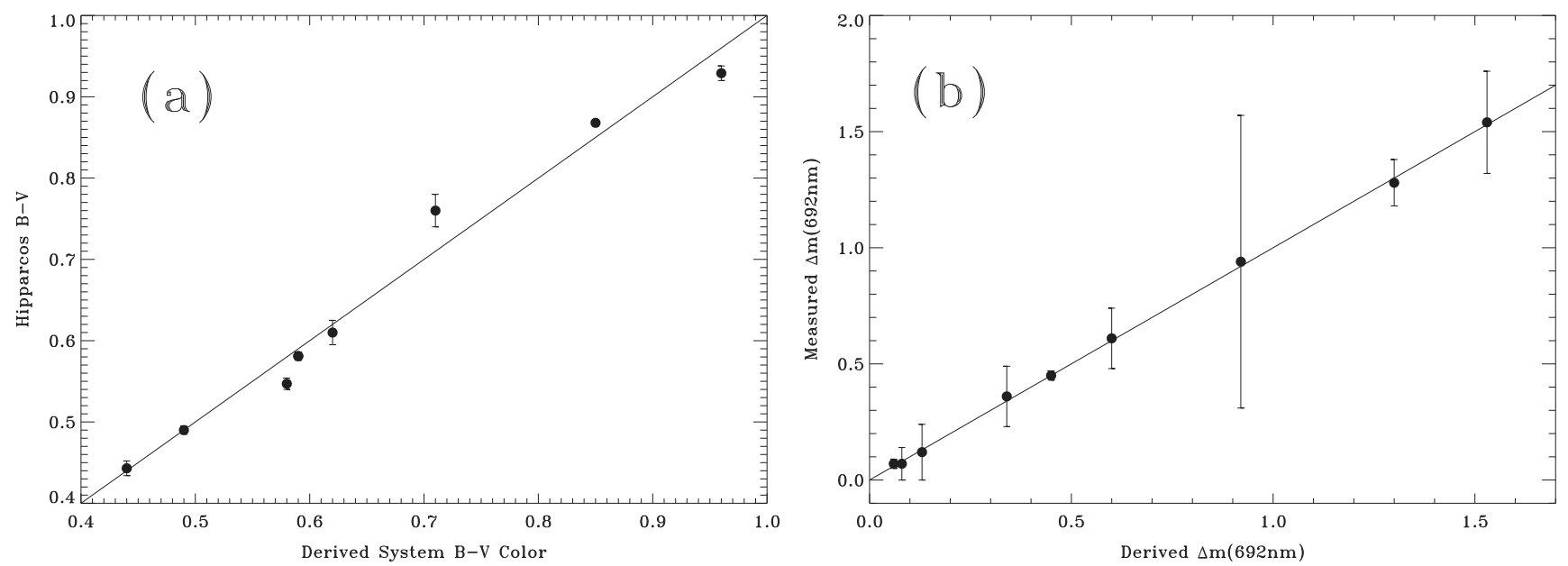

Figure 9. Measured properties of the systems vs. the properties derived from the Pickles-based simulations. (a) $B-V$ color. (b) Magnitude difference at 692 nm. In both cases, the line drawn is $y=x$, indicating that the simulated results are in good agreement with the observed quantities.

Table 6

Mass Comparison for the Systems in Tables 3 and 4

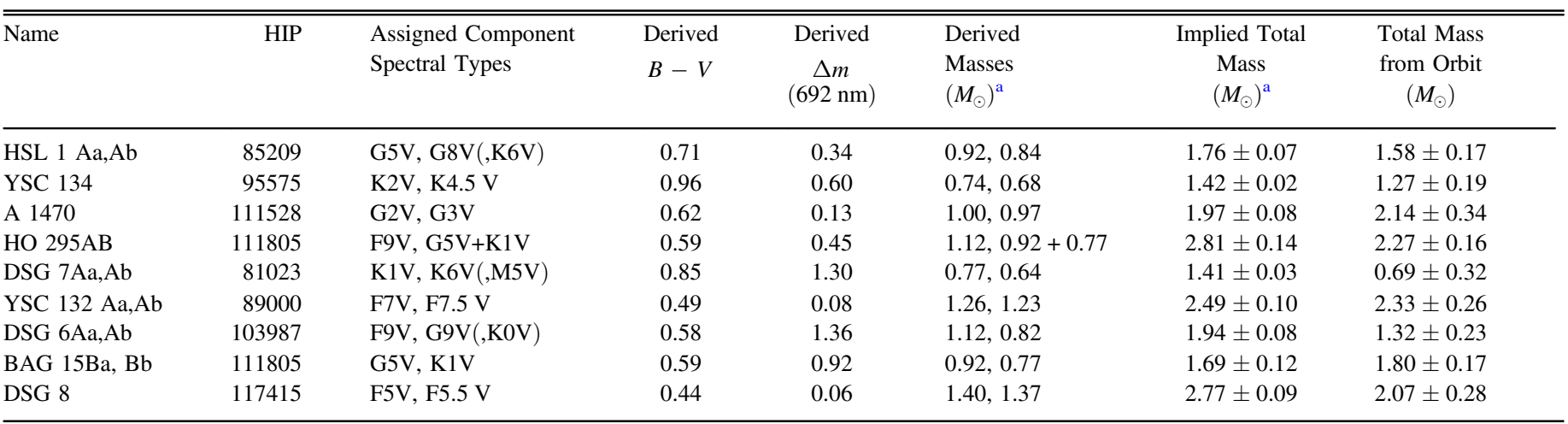

${ }^{\mathrm{a}}$ These columns assume the Solar metal abundance.

We have estimated the uncertainty of the total masses shown by using the scatter in the $B-V$ color. Specifically, we determine a range of spectral types possible for each component within the color uncertainty, we read off the masses corresponding to these high and low estimates of spectral type, and then use them to set the uncertainty interval for the mass of the component. Although the uncertainties in components are not shown in Table 6, we added those in quadrature to obtain an uncertainty estimate in the implied total mass at solar abundance. We also checked the conversion from spectral type to mass using the information provided in the recent work of Boyajian et al. (2012a, 2012b), and found very good agreement with the Schmidt-Kaler reference over the spectral range of interest. Finally, in the last column of Table 6, we show the dynamical total mass estimate using Kepler's harmonic law and the data in Tables 3 and 4. While it is not used in the analysis here, it is worth noting that for the systems that are double-lined spectroscopic binaries, the mass ratios $m_{2} / m_{1}$ implied from Table 6 are in reasonably good agreement from the values implied from the spectroscopic orbits in all cases.

In Figure 10, we plot the theoretical and observed ratio of mass to the solar-abundance mass at the same color, as a function of metal abundance. We have assumed an uncertainty in metallicity of the observed data of $0.1 \mathrm{dex}$ (which is the uncertainty stated in
Holmberg et al. (2009), the source of most of our abundance values). The plot suggests that, within the uncertainty, the points follow the trend expected from the stellar structure calculations. With further work on these and potentially other systems yet to be identified, it should be possible to shrink the vertical error bars in the plot to make more definitive statements concerning the agreement between the observational data and stellar models for a wide range of metallicity.

\section{CONCLUSIONS}

We have presented speckle results on a number of spectroscopic binaries and other nearby stars taken from the Gemini North telescope with the DSSI dual-channel speckle imaging system. These systems are all nearby, but span a range of metal abundance from near the solar value to $[\mathrm{m} / \mathrm{H}]=-1.39$. The precision of the astrometry appears to be in line with that of our previous work at Gemini, namely about 1 mas, and the differential photometry of the observations is precise at the 0.1 magnitude level.

We have used the information presented here together with other astrometry in the literature and the known spectroscopic orbits to obtain high-quality values for the semimajor axis, inclination, and ascending node for nine systems. Using the semimajor axes together with the spectroscopic periods and 


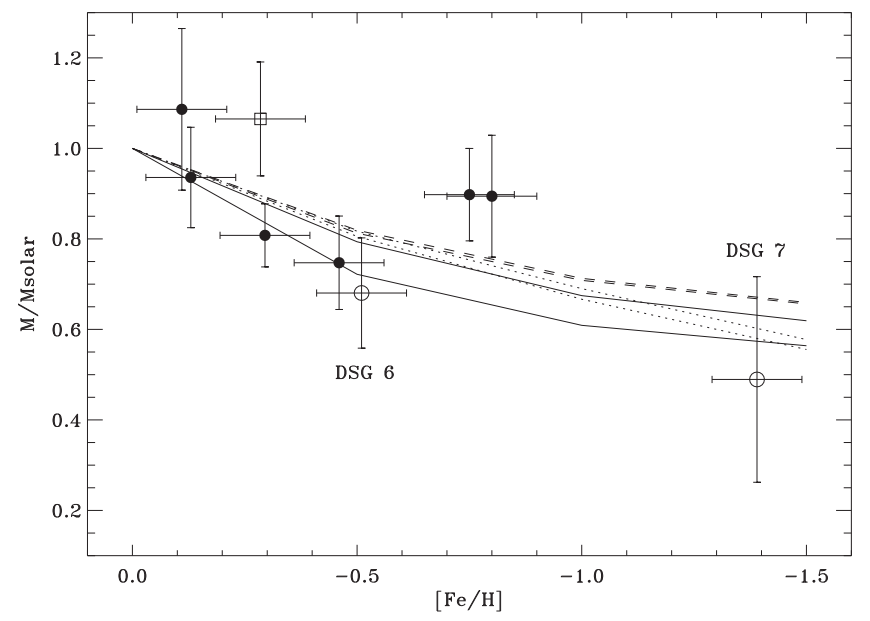

Figure 10. Simulated solar metallicity binaries are constructed with components that match the $B-V$ colors and observed magnitude differences for a set of nine sub-solar metallicity binaries. The ratio of the total mass of each observed binary to the total mass of its (solar-metallicity) simulated counterpart is plotted as a function of observed metallicity. The curves shown are derived from the Spada et al. (2013) stellar models as described in the text; solid lines correspond to a spectral type of F5V $(B-V=0.44)$, dashed curves to $\mathrm{G} 5 \mathrm{~V}(B-V=0.68)$, and dotted lines to $\mathrm{K} 5 \mathrm{~V}(B-V=1.15)$. In all three cases, curves for two ages are shown, $0.1 \mathrm{Gyr}$ and 4.0 Gyr. The singlelined spectroscopic binaries in Tables 3 and 4 are shown as open circles, and the double-lined systems are shown as filled circles. BAG 15Aa,Ab is shown as with an open square; this system is discussed in the text as having a discrepancy between the time of periastron passage as determined from spectroscopy vs. the result from the relative astrometry. The observed trend toward smaller masses as the metallicity decreases matches the prediction from the stellar models.

known parallaxes resulted in total mass estimates. These were compared with theoretical predictions for $\mathrm{F}$ through $\mathrm{M}$ main sequence stars as a function of metallicity. We find that, over this spectral range, our results are so far consistent with the predicted overall trend toward a lower mass at a given spectral type as the metal abundance decreases.

We thank the Kepler Science Office located at the NASA Ames Research Center for providing partial financial support for the upgraded DSSI instrument. It is also a pleasure to thank Steve Hardash, Andy Adamson, Inger Jorgensen, John White, and the entire summit crew for their excellent work in getting the instrument to the telescope and installing it. This work was funded by the Kepler Science Office and NSF grant AST1429015. It made use of the Washington Double Star Catalog maintained at the U.S. Naval Observatory, the SIMBAD database, operated at CDS, Strasbourg, France, and the 9th Catalog of Spectroscopic Orbits of Binary Stars.

\section{REFERENCES}

Balega, I. I., Balega, Y. Y., Hofmann, K.-H., et al. 2002, A\&A, 385, 87 Balega, I. I., Balega, Y. Y., Maksimov, A. F., et al. 2006, BSAO, 59, 20 Balega, I. I., Balega, Y. Y., Maksimov, A. F., et al. 2007, AstBu, 62, 339 Boyajian, T. S., von Braun, K., van Belle, G., et al. 2012a, ApJ, 746, 101 Boyajian, T. S., McAlister, H. A., van Belle, G., et al. 2012b, ApJ, 757, 112 Demarque, P., Woo, J.-H., Kim, Y.-C., \& Yi, S. K. 2004, ApJS, 155, 667 Docobo, J. A., Campo, P., Andrade, M., \& Horch, E. 2013, IAUC, 26, 181 Duquennoy, A. 1987, A\&A, 178, 114

ESA 1997, The Hipparcos and Tycho Catalogues (ESA SP-1200; Noordwijk: ESA)

Freiden, G. A., \& Chaboyer, B. 2012, ApJ, 757, 42

Goldberg, D., Mazeh, T., Latham, D. W., et al. 2002, AJ, 124, 1132

Gratton, R. G., Fusi Pecci, F., Carretta, E., et al. 1997, ApJ, 491, 749

Griffin, R. F. 1999, Obs, 119, 81

Griffin, R. F. 2007, Obs, 127, 113

Halbwachs, J.-L., Arenou, F., Famaey, B., et al. 2012, in SF2A-2012: Proc. Annual Meeting of the French Society of Astronomy and Astrophysics 87, ed. S. Boissier et al. (Paris: Société Française d'Astronomie et d'Astrophysique)

Hartkopf, W. I., Mason, B. D., \& McAlister, H. A. 1996, AJ, 111, 370

Hartkopf, W. I., Mason, B. D., \& Worley, C. E. 2001a, AJ, 122, 3472

Hartkopf, W. I., McAlister, H. A., \& Mason, B. D. 2001b, AJ, 122, 3480

Holmberg, J., Nordström, B., \& Andersen, J. 2009, A\&A, 501, 941

Horch, E. P., Franz, O. G., \& van Altena, W. F. 2006a, AJ, 132, 2478

Horch, E. P., Franz, O. G., Wasserman, L. H., \& Heasley, J. N. 2006b, AJ, 132,836

Horch, E. P., Gomez, S. C., Sherry, W. H., et al. 2011a, AJ, 141, 45

Horch, E. P., Howell, S. B., Everett, M. E., \& Ciardi, D. R. 2012, AJ, 144, 165

Horch, E. P., Howell, S. B., Everett, M. E., \& Ciardi, D. R. 2014, ApJ, 795, 60

Horch, E. P., van Altena, W. F., Howell, S. B., et al. 2011b, AJ, 141, 180

Horch, E. P., Veillette, D. R., Baena Gallé, R., et al. 2009, AJ, 137, 5057

Howell, S. B., Horch, E. P., Everett, M. E., \& Ciardi, D. R. 2012, PASP, 124,1124

Latham, D. W., Mazeh, T., Stefanik, R. P., et al. 1992, AJ, 104, 774

Latham, D. W., Stefanik, R. P., Torres, G., et al. 2002, AJ, 124, 1144

MacKnight, M., \& Horch, E. P. 2004, BAAS, 36, 788

Mason, B. D., Hartkopf, W. I., Holdenried, E. R., \& Rafferty, T. J. 2001b, AJ, 121,3224

Mason, B. D., Wycoff, G. L., Hartkopf, W. I., et al. 2001a, AJ, 122, 3466

Meng, J., Aitken, G. J. M., Hege, K., \& Morgan, J. S. 1990, JOSAA, 7, 1243

Muterspaugh, M. W., Hartkopf, W. I., Lane, B. F., et al. 2010, AJ, 140, 1623

Nordström, B., Mayor, M., Andersen, J., et al. 2004, A\&A, 419, 989

Pickles, A. J. 1998, PASP, 110, 749

Pourbaix, D. 2000, A\&AS, 145, 215

Reid, I. N. 1997, AJ, 114, 161

Schmidt-Kaler, T. 1982, in Landolt-Bärnstein New Series, Group 6, Vol. 2b, Stars and Star Clusters, ed. K. Schaefers \& H.-H. Voigt (Berlin: Springer), 1 Spada, F., Demarque, P., Kim, Y.-C., \& Sills, A. 2013, ApJ, 776, 87 ten Brummelaar, T. A., Mason, B. D., Bagnuolo, W. G., Jr., et al. 1996, AJ, 112,1180

Torres, G., Andersen, J., \& Giménez, A. 2010, A\&ARv, 18, 67

van Leeuwen, F. 2007, A\&A, 474, 653 